University of Nebraska - Lincoln

DigitalCommons@University of Nebraska - Lincoln

2-2009

\title{
Picosecond imaging of low-density plasmas by electron deflectometry
}

\author{
Martin Centurion \\ University of Nebraska-Lincoln, martin.centurion@unl.edu \\ P. Reckenthaeler \\ Max-Planck-Institut für Quantenoptik \\ F. Krausz \\ Max-Planck-Institut für Quantenoptik \\ E. E. Fill \\ Max-Planck-Institut für Quantenoptik
}

Follow this and additional works at: https://digitalcommons.unl.edu/physicscenturion

Part of the Physics Commons

Centurion, Martin; Reckenthaeler, P.; Krausz, F.; and Fill, E. E., "Picosecond imaging of low-density plasmas by electron deflectometry" (2009). Martin Centurion Publications. 5.

https://digitalcommons.unl.edu/physicscenturion/5

This Article is brought to you for free and open access by the Research Papers in Physics and Astronomy at DigitalCommons@University of Nebraska - Lincoln. It has been accepted for inclusion in Martin Centurion Publications by an authorized administrator of DigitalCommons@University of Nebraska - Lincoln. 
This paper was published in Optics Letters 34, 539 (2009) and is made available as an electronic reprint with the permission of OSA. The paper can be found at the following URL on the OSA website: http://www.opticsinfobase.org/ol/abstract.cfm?URI=ol-34-4-539. Systematic or multiple reproduction or distribution to multiple locations via electronic or other means is prohibited and is subject to penalties under law.

\title{
Picosecond imaging of low-density plasmas by electron deflectometry
}

\author{
M. Centurion, ${ }^{1, *}$ P. Reckenthaeler, ${ }^{1}$ F. Krausz, ${ }^{1,2}$ and E. E. Fill ${ }^{1}$ \\ ${ }^{1}$ Max-Planck-Institut für Quantenoptik, D-85748 Garching, Germany \\ ${ }^{2}$ Ludwig-Maximilians-Universität München, Am Coulombwall 1, D-85748 Garching, Germany \\ *Corresponding author: martin.centurion@mpq.mpg.de
}

Received October 3, 2008; revised December 10, 2008; accepted December 19, 2008;

posted January 21, 2009 (Doc. ID 102363); published February 13, 2009

We have imaged optical-field ionized plasmas with electron densities as low as $10^{13} \mathrm{~cm}^{-3}$ on a picosecond timescale using ultrashort electron pulses. Electric fields generated by the separation of charges are imprinted on a $20 \mathrm{keV}$ probe electron pulse and reveal a cloud of electrons expanding away from a positively charged plasma core. Our method allows for a direct measurement of the electron energy required to escape the plasma and the total charge. Simulations reproduce the main features of the experiment and allow determination of the energy of the electrons. (c) 2009 Optical Society of America

OCIS codes: $140.3440,320.7100$

Optical-field ionization (OFI) continues to be of high experimental and theoretical interest. Owing to their unique properties, OFI plasmas have gained applications in various fields, such as inertial confinement fusion [1], generation of high-energy ions [2,3], x-ray emission [4,5], and x-ray lasers [6-10]. The parameters of OFI plasmas can be determined by a variety of diagnostic methods. The electron temperature can be measured by Thomson scattering [11]. The ionization stage can be determined by ion spectrometry [12] and by x-ray spectroscopy [4]. The time-resolved plasma-density profile has been measured using optical interferometry, holography, and Moiré deflectometry [13-15].

In a recent paper we demonstrated a new technique of plasma diagnostics by deflecting electrons in the fields of the plasma [16], where we observed that a small fraction of electrons acquires enough energy from the laser to escape the plasma. In this Letter we apply this technique to plasmas with very low density. In this regime we measure very different features, most notably, the minimum electron energy required to escape the plasma is much lower, resulting in most of the electrons flying away and leaving behind a positively charged plasma core.

The electron gun used in our experiments has been described in a previous publication [16]. The plasma is generated by focusing a laser pulse ( $1 \mathrm{~mJ}$ energy, $50 \mathrm{fs}$ duration) inside a chamber filled with nitrogen at a uniform low density. The laser is focused to a diameter of $20 \mu \mathrm{m}$ and intensity of $4 \times 10^{15} \mathrm{~W} / \mathrm{cm}^{2}$. The probe electron pulse is generated using a small fraction of the laser pulse energy to trigger electron emission from a photocathode. The electrons are accelerated to $20 \mathrm{keV}$ in a static field before traversing the OFI plasma in a direction perpendicular to the laser beam. After interacting with the plasma the electron beam impinges on a scintillating layer that is fiber coupled to a CCD camera. An optical delay stage is used to adjust the relative delay between laser and electron pulses.

Figure 1 shows the distortion of the electron beam due to the plasma, at a relative delay of 100 ps. Fig- ures 1(a) and 1(b) show the electron beam as recorded on the CCD without and with the plasma, respectively, for a gas pressure of $2.6 \times 10^{-4}$ mbar. The path of the laser beam shows up as a narrow line with increased electron flux, surrounded by a depleted region. Figures 1(c)-1(f) show cross sections of the electron beam [along the dotted line in Fig. 1(b)] for increasing gas density $\left(8.6 \times 10^{-5}\right.$ mbar, 2.6 $\times 10^{-4}$ mbar, $1.1 \times 10^{-3}$ mbar, and $5.3 \times 10^{-3}$ mbar, respectively). The line results from focusing of the electron beam by a positively charged plasma cylinder (the laser ionizes a cylindrical volume as it propagates through the gas). The positive charge at the center is screened by an expanding cloud of electrons; thus only a fraction of the probe electrons are deflected [Fig. 1(g)]. For higher gas density the charge at the center increases, leading to overfocusing of the electron beam (the beam goes through a focus before reaching the CCD). The pattern becomes weaker for lower pressures and is only barely visible for pressures below $5 \times 10^{-5}$ mbar (not shown). At an intensity of $4 \times 10^{15} \mathrm{~W} / \mathrm{cm}^{2}$ the classical over the barrier model [12] predicts four electrons being ionized from each nitrogen molecule. The electron energy generated by a linearly polarized laser is relatively low [17], but electrons will still be able to leave the central plasma core and generate electric fields.

The effect of the outgoing electrons as a function of time is displayed in Fig. 2 for a nitrogen pressure of $6.5 \times 10^{-5}$ mbar. Each vertical line in the figure corresponds to a cross section [same as for Figs. 1(c)-1(f)] for a specific time delay. The red line along the center represents increased counts on the detector, while the blue area corresponds to the depleted region (decreased counts) around the focus as seen also in Figs. 1(c)-1(f). The boundary of the dark blue expanding region corresponds to the maximum distance from which electrons are deflected toward the center. This boundary expands with a velocity of 1.1 $\times 10^{6} \mathrm{~m} / \mathrm{s}$, corresponding to an electron energy of $3.4 \mathrm{eV}$. We can thus conclude that plasma electrons with energy $\geqslant 3.4 \mathrm{eV}$ can escape from the plasma core. Below we use a theoretical model to compare 
(a)

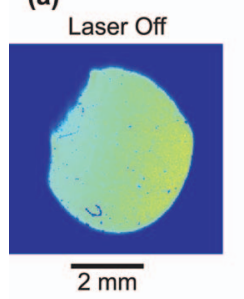

(b)
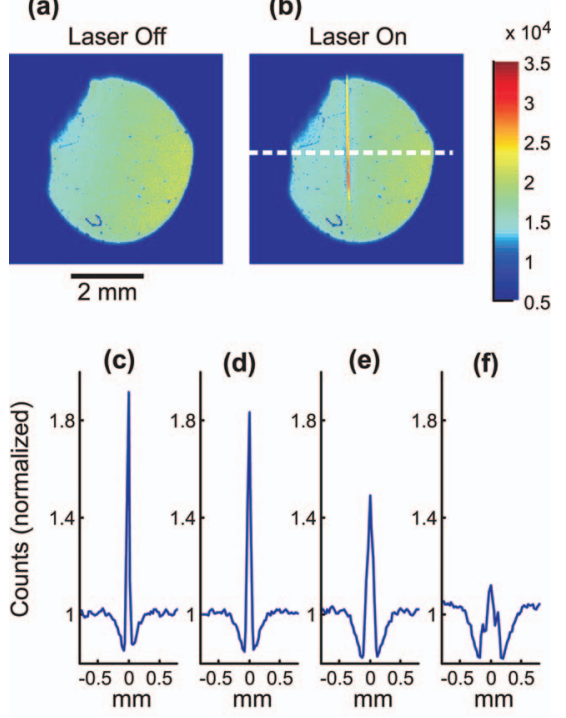

(g)

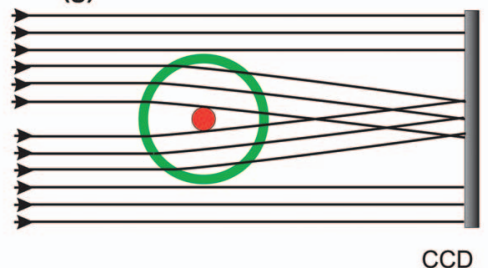

Fig. 1. Deflection of electrons owing to plasma fields at a time delay of $100 \mathrm{ps}$. (a), (b) Electron-beam pattern recorded on the CCD without plasma and with plasma, respectively, for a gas density of $2.6 \times 10^{-4} \mathrm{mbar}$. The scale is the same for both images, and the color bar denotes the number of counts per pixel on the CCD. (c)-(f) Cross sections of the electron-beam pattern [along the dotted line in Fig. 1(b)] for gas densities of $8.6 \times 10^{-5}$ mbar, 2.6 $\times 10^{-4}$ mbar, $1.1 \times 10^{-3} \mathrm{mbar}$, and $5.3 \times 10^{-3} \mathrm{mbar}$, respectively, normalized to the number of counts on the undisturbed electron beam. (g) Sketch of a few representative trajectories of the probe beam electrons traversing a plasma with cylindrical symmetry. The red circle in the center represents a positively charged core, and the green ring represents a cloud of electrons escaping from the plasma core. The area outside of the green ring is completely screened from the positive charges; i.e., it is field free. These trajectories would generate on the detector a focused line surrounded by a depleted region.

with experiment and extract the temperature of the plasma electrons.

The analysis is carried out in cylindrical geometry. Experimentally, the laser pulse ionizes the nitrogen in a cylindrical volume with diameter of $20 \mu \mathrm{m}$ and length on the order of $1 \mathrm{~mm}$. A central charge with a line density of $\mathrm{N}^{+}$ions per meter generates a radial electric field that deflects beam electrons toward the center by an angle $\theta=e N^{+} / 4 U \varepsilon_{0}$, where $U$ is the electron energy in $\mathrm{eV}, e$ is the elementary electric charge, and $\varepsilon_{0}$ the vacuum permittivity. The deflection angle is independent of the distance from the center, similar to a cylindrical biprism. This simple analytical treatment, however, does not take into account that plasma electrons partially screen the central charge. At early times all electrons are still close to the center, and most of the surrounding volume is screened;

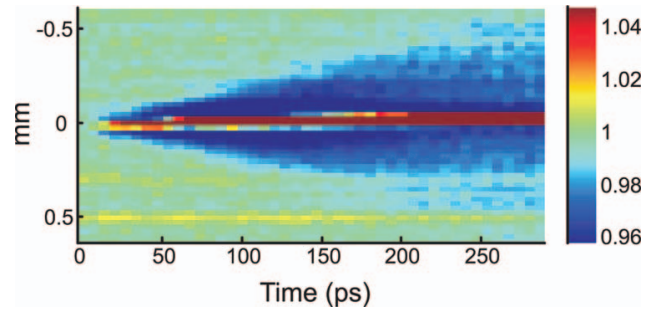

Fig. 2. Evolution of the plasma fields. The vertical axis corresponds to a cross section through the plasma line, as shown in Fig. 1(b). The horizontal axis is the pump-probe delay. The color scale corresponds to the number of counts per pixel on the CCD, normalized to the number of counts on the undisturbed electron beam.

thus the electron beam is depleted only in a region close to the plasma core. The positive ions remain fixed inside the plasma cylinder throughout the duration of the experiment owing to their large mass. As the plasma electrons move away from the center the screening decreases, resulting in the expansion of the depleted region and an increase in the intensity of the focused line.

On the basis of the model described above we have carried out simulations coupling the result of a hydrodynamic plasma code [18] (which solves the equations of motion of the electrons in Lagrangian coordinates) to the general particle tracer code for calculating electron-beam propagation [19]. The temporal evolution of the peak intensity of the focused line is presented in Fig. 3. The experimental values are compared with simulations for $20 \mathrm{eV}, 30 \mathrm{eV}$, and $40 \mathrm{eV}$. For an electron energy of $20 \mathrm{eV}$ the simulation shows a reflux of electrons to the plasma core after $150 \mathrm{ps}$, which results in a sudden drop of the peak intensity. An exponential electron energy distribution with a temperature of $30 \mathrm{eV}$ gave best agreement with experiment. The local minimum in the experimental data around $150 \mathrm{ps}$ is an artefact owing to the limited number of pixels that sample the peak.

Figure 4 shows cross sections of the experimental and simulated electron-beam profiles. The simulations accurately reproduce the experimental curves,

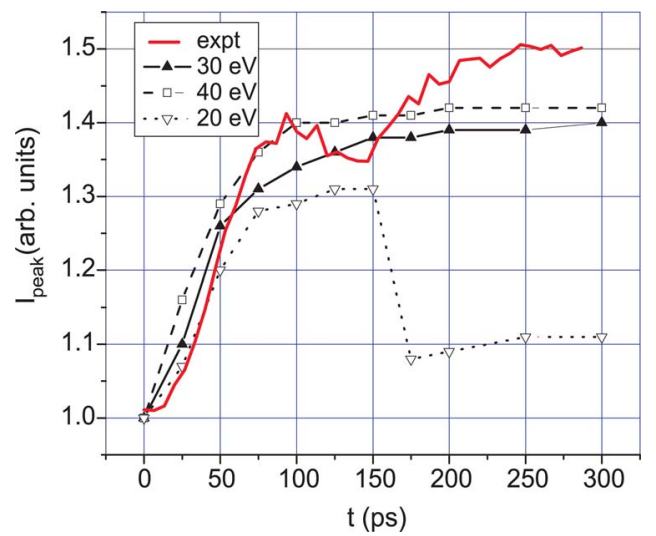

Fig. 3. (Color online) Peak intensity as a function of delay time. The intensity is normalized to the intensity of the undisturbed electron beam. The initial electron density is $10^{13} \mathrm{~cm}^{-3}$. The figure compares experimental data with simulations assuming three different electron temperatures. 
(a) Experiment

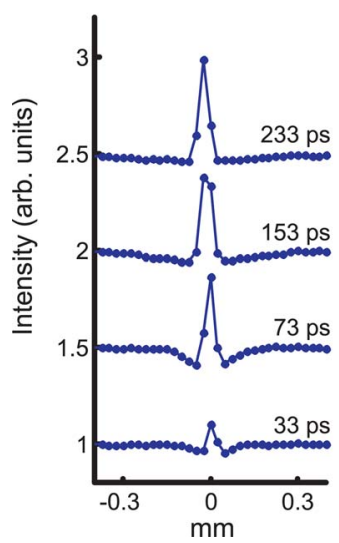

(b) Theory

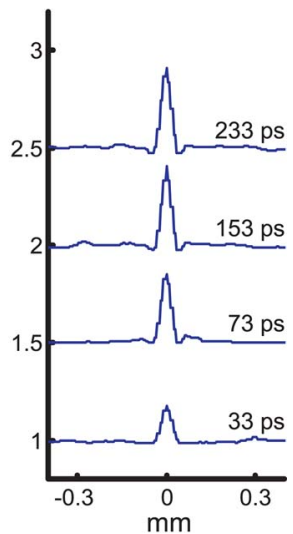

Fig. 4. (Color online) Cross sections through the center of the focused line on the CCD for different time delays. (a) Experimental results, with parameters as in Fig. 2. (b) Results from the simulation. The intensity is normalized to the intensity of the undisturbed electron beam. The plots for different delay times are displaced vertically for visual clarity.

both in the temporal evolution and the amplitude of the peaks. The only adjustable parameter in the simulations is the electron temperature. The calculated exponential electron temperature of $30 \mathrm{eV}$, along with the experimental observation that electrons with energy $\geqslant 3.4 \mathrm{eV}$ escape from the plasma core, allow us to calculate that roughly $90 \%$ of the ionized electrons escape, leaving behind a highly charged plasma.

In conclusion, we have observed a cloud of electrons expanding away from a positively charged plasma, with as much as $90 \%$ of the electrons escaping for an initial plasma density of $7 \times 10^{12} \mathrm{~cm}^{-3}$. In contrast, in a previous study [16] we have observed that for plasma densities of $10^{18} \mathrm{~cm}^{-3}$ only a small fraction $\left(5 \times 10^{-4}\right)$ of the electrons manage to escape the plasma. Electrons are accelerated by the laser, and in the case of low density the plasma fields are not strong enough to trap them. From our experiments we have directly measured an escape energy of $3.4 \mathrm{eV}$ for the electrons, and an electron temperature of $30 \mathrm{eV}$ was obtained by comparing the experimental results with simulations.

This work was supported by Deutsche Forschungsgemeinschaft (DFG) under contract SFB Transregio 6039 and by the DFG Cluster of Excellence "Munich
Centre for Advanced Photonics.” M. Centurion was supported by a research fellowship from the Alexander von Humboldt Foundation.

\section{References}

1. T. Ditmire, J. Zweiback, V. P. Yanovsky, T. E. Cowan, G. Hays, and K. B. Wharton, Nature 398, 489 (1999).

2. T. Ditmire, J. W. G. Tisch, E. Springate, M. B. Mason, N. Hay, R. A. Smith, J. Marangons, and M. H. R. Hutchinson, Nature 386, 54 (1997).

3. M. S. Wei, S. P. D. Mangles, Z. Najmudin, B. Walton, A. Gopal, M. Tatarakis, A. E. Dangor, E. L. Clark, R. G. Evans, S. Fritzler, R. J. Clarke, C. Hernandez-Gomez, D. Neely, W. Mori, M. Tzoufras, and K. Krushelnik, Phys. Rev. Lett. 93, 155003 (2004).

4. E. Fill, S. Borgström, J. Larsson, T. Starczewski, C.-G. Wahlström, and S. Svanberg, Phys. Rev. E 51, 6016 (1995).

5. A. McPherson, T. S. Luk, B. D. Thompson, K. Boyer, and C. K. Rhodes, Appl. Phys. B 57, 337 (1993).

6. P. Amendt, D. C. Eder, and S. C. Wilks, Phys. Rev. Lett. 66, 2589 (1991).

7. B. E. Lemoff, G. Y. Yin, C. L. Gordon III, C. P. J. Barty, and S. E. Harris, Phys. Rev. Lett. 74, 1574 (1995).

8. Y. Nagata, K. Midorikawa, S. Kubodera, M. Obara, H. Tashiro, and K. Toyoda, Phys. Rev. Lett. 71, 3774 (1993).

9. S. Sebban, T. Mocek, D. Ros, L. Upcraft, P. Balcou, R. Haroutunian, G. Grillon, B. Rus, A. Klisnick, A. Carillon, G. Jamelot, C. Valentin, A. Rousse, J. P. Rousseau, L. Notebaert, M. Pittman, and D. Hulin, Phys. Rev. Lett. 89, 253901 (2002).

10. J. P. Godded, S. Sebban, A. S. Morlens, J. Gautier, J. P. Rousseau, F. Burgy, P. Zeitoun, C. Valentin, C. Hauri, G. Maynard, A. Boudaa, H. Merdji, T. Mocek, M. Kozlova, and K. Jakubczak, Opt. Lett. 32, 1498 (2007).

11. T. E. Glover, T. D. Donnelly, E. A. Lipman, A. Sullivan, and R. W. Falcone, Phys. Rev. Lett. 73, 78 (1994).

12. S. Augst, D. D. Meyerhofer, D. Strickland, and S. L. Chin, J. Opt. Soc. Am. B 8, 858 (1991).

13. S. Tzortzakis, B. Prade, M. Franco, and A. Mysyrowicz, Opt. Commun. 181, 123 (2000).

14. M. Centurion, Y. Pu, D. Psaltis, and T. W. Hänsch, Opt. Lett. 29, 772 (2004).

15. M. Dunne, T. Afshar-Rad, J. Edwards, A. J. MacKinnon, S. M. Viana, O. Willi, and G. Pert, Phys. Rev. Lett. 72, 1024 (1994).

16. M. Centurion, P. Reckenthäler, S. Trushin, F. Krausz, and E. Fill, Nat. Photonics 2, 315 (2008).

17. P. B. Corkum, N. H. Burnett, and F. Brunel, Phys. Rev. Lett. 62, 1259 (1989).

18. E. E. Fill, Phys. Plasmas 12, 052704-1 (2005).

19. http://www.pulsar.nl/gpt. 